\title{
DISCAPACIDAD Y ACCESIBILIDAD UNIVERSAL. LA UNIÓN PERFECTA.
}

\author{
Guillermo López Cala \\ Servicio Andaluz de Salud \\ Eva Sotomayor Morales \\ María Luisa Grande Gascón \\ María José Calero García \\ Universidad de Jaén (España)
}

\begin{abstract}
Resumen. España es un país con un modelo de atención y bienestar social basado en los derechos humanos, cuyo objetivo entre otros es la no discriminación y la igualdad de oportunidades.

Tradicionalmente las personas con discapacidad o diversidad funcional han estado sujetas de prejuicios, encontrándose en muchas circunstancias en situación de vulnerabilidad social, sufriendo discriminación y exclusión con una disminución en su calidad de vida y con problemas para acceder a determinados servicios, productos y lugares. Por lo tanto, creemos que es fundamental tener en cuenta que hay que mejorar constantemente y de forma inmediata y exponencial, la situación de los entornos productos y servicios destinados a combatir o paliar de alguna manera los distintos grados de discapacidad. Y esa mejora puede aparecer desde la aplicación y desarrollo del concepto Accesibilidad Universal.

Es necesario por tanto reflexionar sobre si nuestra sociedad está siendo capaz de desarrollar y realizar acciones e intervenciones administrativas, legislativas, políticas, económicas, educativas y de información y concienciación social, etc., en lo concerniente a la Accesibilidad Universal y la Diversidad Funcional. Por otra parte, las deficiencias de nuestra sociedad y la falta de solidaridad e implementación en la puesta en marcha y mantenimiento de las políticas orientadas hacia las personas con diversidad funcional es a veces un importante hándicap para el correcto cuidado de estos. Esto hace que nuestra sociedad no aparezca como justa, equitativa, global, responsable y garante de los derechos fundamentales de todos los ciudadanos por igual ante cualquier tipo de discapacidad.
\end{abstract}

Palabras clave: diversidad funcional, discapacidad, accesibilidad universal, exclusión social.

\section{DISABILITY AND UNIVERSAL ACCESSIBILITY. THE PERFECT UNION.}

\begin{abstract}
Spain is a country with a model of care and social welfare based on human rights, whose objective among others is non-discrimination and equal opportunities. Traditionally, people with disabilities or functional diversity have been subject to prejudice, being in many circumstances in a situation of social vulnerability, suffering discrimination and exclusion with a decrease in their quality of life and problems to access certain services, products and places. Therefore, we believe that it is essential to take into account that we must constantly and immediately and exponentially improve the situation of the environment products and services intended to combat or alleviate in some way the different degrees of disability. And that improvement can appear from the application and development of the Universal Accessibility concept. It is therefore necessary to reflect on whether our society is able to develop and carry out administrative, legislative, political, economic, educational and informational actions and interventions, and social awareness, etc., concerning Universal Accessibility and Funtional Diversity. On the other hand, the deficiencies of our society and the lack of solidarity and implementation in the implementation and maintenance of policies oriented towards people with functional diversity is sometimes an important handicap for the proper care of these. This makes our society not appear as fair,
\end{abstract}


equitable, global, responsible and guarantor of the fundamental rights of all citizens alike to any type of disability .

Keywords: Functional diversity, disability, universal accessibility, social exclusion.

\section{DEFICIÊNCIA E ACCESIBILIDADE UNIVERSAL.}

\section{A UNIÃU PERFEITA.}

Resumo. Espanha é um país com um modelo de cuidado e bem-estar baseado nos direitos humanos, cujo objetivo, entre outros é não-discriminação e igualdade de oportunidades. Tradicionalmente, as pessoas com deficiência ou diversidade funcional tenham sido objecto de preconceito, sendo, em muitas circunstâncias socialmente vulneráveis, sofrendo discriminação e exclusão com uma diminuição da sua qualidade de vida e problemas para acessar certos serviços, produtos e lugares. Portanto, nós acreditamos que é essencial observar que deve ser constantemente e imediatamente e de forma exponencial melhorar a situação dos produtos e serviços ambientais para combater ou atenuar de alguma forma diferentes graus de deficiência. E esta melhoria pode aparecer a partir da aplicação e desenvolvimento de Acessibilidade Universal conceito. Por isso, é necessário considerar se a nossa sociedade é ser capaz de desenvolver e implementar ações e informações administrativas, legislativas, político, econômico, educacional e social e intervenções de sensibilização, etc., relativos à Accessibilidade Universal e Diversidade Funcional. Além disso, as deficiências da nossa sociedade e a falta de solidariedade e implementação na implementação e manutenção de políticas para as pessoas com deficiência às vezes é uma grande desvantagem para o bom atendimento destes. Isso faz com que a nossa sociedade não aparece como justa, equitativa, global, responsável e garante dos direitos fundamentais de todos os cidadãos igualmente a todos os tipos de deficiência.

Palavras-chave: diversidade funcional, deficiência, acessibilidade universal, a exclusão social.

\section{Introducción}

En muchas situaciones, pero sobre todo a partir del envejecimiento de las personas, se derivan situaciones de aumento de la discapacidad y vulnerabilidad. La discapacidad tarde o temprano nos afecta a todos en sus diferentes escalas. Ya sea temporal por una simple torcedura de tobillo o de forma permanente producidas por enfermedades graves invalidantes o accidentes, etc.

El Informe Mundial sobre Discapacidad de la OMS (2011), donde insiste en que la discapacidad es una cuestión de derechos humanos, por cuanto las personas con discapacidad experimentan situaciones de desigualdad y de violación de la dignidad, en ocasiones, por prejuicios y abusos, y tienen serias dificultades para lograr su autonomía personal.

Según la encuesta de Discapacidad, Autonomía personal y situaciones de Dependencia (EDAD) del año 1999, el total de población en España afectada de alguna discapacidad entre el intervalo de edad de 6 a 64 años, de ambos sexos era de 1.405 .992 personas, mientras que en el año 2008, perteneciente al Instituto Nacional de estadística (INE), la misma encuesta arroja los siguientes datos:

El número de personas con discapacidad en España alcanza los 3,8 millones, lo que suponía el 8,5\% de la población. De ellas un total de 608.000 personas con discapacidad viven solas en su hogar. Es decir, estamos hablando de más del doble de personas discapacitadas en apenas 9 años. Lo cual es tremendamente significativo, y es una tendencia que se prevé que se mantendrá a lo largo de los años, además agravada 
por el envejecimiento progresivo de la población no solo a nivel de España sino mundial.

De los 3,8 millones de personas con discapacidad funcional de los que hablábamos, 1.390 .000 personas no pueden realizar alguna de las actividades básicas de la vida diaria (ABVD) sin ayuda. Lo cual nos avanza aun más en la importancia de ahondar en la preparación de los conocimientos y la mejora de la calidad de vida de las personas discapacitadas actuales y los futuras.

Las ABVD son las tareas más elementales de la persona, que le permiten desenvolverse con un mínimo de autonomía e independencia, tales como: el cuidado personal, las actividades domésticas básicas, la movilidad esencial, reconocer personas y objetos, orientarse, entender y ejecutar órdenes o tareas sencillas. (Artículo 2. Ley 39/2006, de 14 de diciembre, de Promoción de la Autonomía Personal y Atención a las personas en situación de dependencia. Jefatura del Estado. BOE 299, de 15 de diciembre de 2006)

Otra definición de las ABVD es "el conjunto de actividades primarias de la persona, encaminadas a su autocuidado y movilidad y a la capacidad de entender y ejecutar órdenes y tareas sencillas, que le dotan de autonomía e independencia elementales y le permiten vivir sin precisar ayuda continua de otros. Entre ellas se incluyen actividades como comer, controlar esfínteres, usar el retrete, vestirse, bañarse, trasladarse, deambular, etc." (www.plusesmas.com. Consultada la web en febrero de 2017)

Por otro lado, datos del año 2014 nos indican que el número de personas en España con un grado de discapacidad igual o mayor al 33\%, es de 2.813.592, de las que 1.410.310 (el 50,12 \%) son mujeres y 1.403.282 (el 49,88\%) son hombres (IMSERSO, 2016). Por tanto, de estos datos concluimos que el aumento de la población de personas discapacitadas es muy importante en nuestro país habiéndose duplicado en pocos años.

El objetivo general de este trabajo es doble:

- $\quad$ Poner de manifiesto que es fundamental trabajar en pro del aumento y la calidad de la Accesibilidad Universal para la mejora de las oportunidades y calidad de vida de las personas discapacitadas y mayores.

- Aportar algunas propuestas, ideas y posibles soluciones que mejoren el planteamiento anterior en diversos aspectos, productos o servicios de nuestra sociedad.

Para abordar estos objetivos, es necesario conocer la relación entre Discapacidad o Diversidad Funcional y Accesibilidad Universal, conceptos vinculados hoy en día a la calidad de vida de millones de personas discapacitadas de todo tipo y a la observancia de altos niveles de vulnerabilidad y exclusión social.

En concreto, en primer lugar, recordamos el concepto de Discapacidad, así como las posibles barreras existentes que perjudican a todos pero especialmente a las personas discapacitadas y mayores, y de Accesibilidad Universal y su importancia fundamental para la mejora de la situación de las personas discapacitadas y mayores a cualquier nivel.

Nos referimos a conceptos recogidos en el Libro Verde de la Accesibilidad editado por el IMSERSO, el Concepto Europeo de Accesibilidad, o el Libro Blanco de 
la Accesibilidad de ACCEPLAN ${ }^{1}$, éste último fue el borrador del I Plan Nacional de Accesibilidad 2004-2012 en nuestro país, y otra diversa literatura referente a clasificaciones obre tipos de barreras. Igualmente nos referimos a los llamados criterios DALCO de la Agencia Española de Normalización (AENOR).

\section{El concepto de discapacidad}

Comenzaremos introduciendo el concepto de discapacidad, siendo éste un término general que abarca las deficiencias (OMS, 2011), las limitaciones de la actividad y las restricciones de la participación.

Las deficiencias son problemas que afectan a una estructura o función corporal; las limitaciones de la actividad son dificultades para ejecutar acciones o tareas, y las restricciones de la participación son problemas para participar en situaciones vitales. Todos estos son problemas se ven aumentados con la falta de accesibilidad en diferentes vertientes o formas.

El término discapacidad significa una deficiencia física, mental o sensorial, ya sea de naturaleza permanente o temporal, que limita la capacidad de ejercer una o más actividades esenciales de la vida diaria, que puede ser causada o agravada por el entorno económico y social (Muñoz, 2010)

Según la Organización de Naciones Unidas (ONU), División de Política Social y Desarrollo (2015), existen más de 1000 millones de personas con discapacidad en el mundo.

“...tenemos el deber moral de eliminar los obstáculos a la participación y de invertir fondos y conocimientos suficientes para liberar el inmenso potencial de las personas con discapacidad. Los gobiernos del mundo no pueden seguir pasando por alto a los cientos de millones de personas con discapacidad a quienes se les niega el acceso a la salud, la rehabilitación, el apoyo, la educación y el empleo, y a los que nunca se les ofrece la oportunidad de brillar". (Profesor Stephen W. Hawking, en el prólogo del primer Informe Mundial sobre la Discapacidad. OMS, 2011.)

La Organización Mundial de la Salud, clasifica los tipos de discapacidad que podemos encontrar en (CIE10/OMS): Osteoarticulares, de los sistemas nervioso y muscular (neuromusculares), visuales, auditivas, expresivas, intelectual, mental, de los órganos internos y de la piel, mixta y otras.

Según la información que se recoge en la Base Estatal de Datos de Personas con valoración del Grado de Discapacidad, relativa al histórico de las personas que han solicitado el reconocimiento de la situación con discapacidad, de las distintas Comunidades Autónomas que conforman el Estado Español, incluidas las ciudades autónomas de Ceuta y Melilla, a fecha 31 de diciembre de 2015, arroja la cifra absoluta (con la consideración de personas con discapacidad) de 2.998.591, de las cuales 1.492.946 son hombres y 1.505.645 son mujeres.

Si a esta cifra le añadimos el número de personas mayores de 65 años, según los datos del Padrón continuo del Instituto Nacional de Estadística, a 1 de enero de 2016,

\footnotetext{
${ }^{1}$ ACCEPLAN: Es un equipo técnico multidisciplinar nacido en la Universidad Autónoma de Barcelona el año 2000 y dedicado a la consultoría, investigación y formación para la mejora de la accesibilidad en el medio urbano, la edificación y el transporte.
} 
cifra que se eleva a 8.657 .705 de personas, un 18,4\% sobre un total de población de 46.557.008 de españoles, obtendríamos un total de 11.656.344 personas susceptibles de sufrir alguna situación de inaccesibilidad mayor que el resto de la población total española referida.

El 22 de mayo de 2001, en la 54 ${ }^{\mathrm{a}}$ Asamblea de la OMS, se aprobó la nueva versión de la Clasificación Internacional del funcionamiento, la Discapacidad y la Salud, con las siglas CIF.

La clasificación habla de funcionamiento como término genérico para designar todas las funciones y estructuras corporales, la capacidad de desarrollar actividades y la posibilidad de participación social del ser humano, la discapacidad de igual forma como término genérico que recoge las deficiencias en las funciones y estructuras corporales, las limitaciones en la capacidad de llevar a cabo actividades y las restricciones en la participación social del ser humano. Y la salud como elemento clave que relaciona lo anteriormente expuesto. (Web Cruz Roja Española. Consultado en mayo de 2017)

La discapacidad puede ser de tipo físico, psíquico o psicosocial, sensorial e intelectual o mental y a la par podría ser una discapacidad de tipo temporal o permanente. (2013):

En general se distinguen 6 categorías de discapacidad según Geiser y Chervin

- Discapacidad motora: cubre todos los trastornos que pueden causar deterioro parcial o total de las habilidades motoras incluyendo la parte superior y/o inferior del cuerpo (dificultades para caminar, dificultad en el mantenimiento o el cambio de posición, y en la manipulación o la realización de determinadas acciones). Algunas discapacidades motoras de origen cerebral también pueden causar dificultades para expresarse, sin deterioro de la capacidad mental.

- $\quad$ Discapacidad visual: se refiere a personas ciegas y también, en la mayoría de los casos, a las personas con trastornos severos de la visión. En algunas profesiones una persona daltónica puede ser reconocida como una persona con discapacidad.

- Discapacidad auditiva: la pérdida de audición total es poco frecuente pues, al igual que con la discapacidad visual, la mayoría de las personas con discapacidad auditiva tienen "audición residual" que puede ser mejorada gracias al impulso de los audífonos.

- Discapacidad intelectual: representa una dificultad en la comprensión y una limitación de la velocidad de las funciones mentales en términos de la comprensión, el conocimiento y la percepción. Estas discapacidades ocurren en diferentes grados y pueden ser perjudiciales para el proceso de retención de conocimientos, la atención, la comunicación, la autonomía social y profesional, la estabilidad y el comportamiento emocional.

- Discapacidad psíquica: no es posible una definición comprensible, especialmente porque la terminología psiquiátrica (neurosis, psicosis...) sólo la dominan los psiquiatras. Sin embargo, podemos decir que las personas con dificultades psíquicas sufren de un malestar que se puede traducir a veces, en conductas perturbadoras para los demás porque son diferentes de las costumbres y hábitos habituales.

- Enfermedades incapacitantes: todas las enfermedades respiratorias, cardiovasculares, digestivas y parasitarias (p.e. cáncer, SIDA, DM, hemofilia 
hipertiroidismo) pueden entrañar deficiencias o limitaciones en diversos grados.

La enfermedad puede ser temporal, permanente o progresiva.

Las situaciones de discapacidad en mayor o menor medida nos llegan a casi todos en algún momento de nuestras vidas, cuanto mayor eres, mayor riesgo se tiene de padecer algún tipo o grado de discapacidad de cualquier tipo. Convirtiéndose esa cada vez mayor población mundial en personas más vulnerables e incluso dependiendo de la situación podríamos llegar a sentirnos excluidos socialmente.

Una de los cauces para llegar a conseguir esto, creemos que es la mejora en la implementación y desarrollo de la Accesibilidad Universal, que podría solucionar o paliar en gran medida estas situaciones, como decimos de auténtico agravio comparativo y de incumplimiento social de los derechos fundamentales de las personas.

\section{La Accesibilidad Universal}

Cualquier persona con dificultades en su movilidad y/o entendimiento intelectual, se enfrenta diariamente a una verdadera carrera de obstáculos que el resto de las personas usuarias no vemos en la mayoría de las ocasiones.

En todos estos casos y situaciones planteadas, la persona puede tener problemas de accesibilidad. Para mejorar, solucionar y facilitar la accesibilidad, vamos a trabajar simplemente en la línea de reflejar la necesidad de que la accesibilidad sea pieza fundamental en la consecución de un objetivo final que debe ser la mejora de las situaciones de discapacidad gracias al conocimiento de la Accesibilidad Universal.

España debe ser un país modelo en la atención y la mejora y cumplimiento del bienestar social basado en los derechos humanos, evitando discriminar a una importante parte de la población, que en ocasiones se encuentran en situaciones de vulnerabilidad e incluso exclusión social.

Tal como se indica en el Libro Verde de la Accesibilidad, editado por el IMSERSO $^{2}$ (Instituto de Migraciones y Servicios Sociales),

"el término accesibilidad, proviene de 〈〈acceso〉>, acción de llegar y acercarse, o bien entrada o paso. Aplicado al uso del espacio o de los objetos y tecnologías, y especialmente con relación a ciertos colectivos de población con dificultades funcionales, la palabra adquiere un matiz menos neutro, más expresivo de los beneficios que se derivan de la interacción con el entorno o con otras personas"

Según el Concepto Europeo de Accesibilidad ${ }^{3}$, ésta es una característica básica del entorno construido, es la condición que posibilita llegar, entrar, salir y utilizar las casas, las tiendas, los teatros, los parques y los lugares de trabajo. Y permite a las personas participar en las actividades sociales y económicas para las que se han concebido. De esto se desprende que, cuando exista una falta de accesibilidad, por ejemplo, con barreras físicas, esa persona sufrirá algún grado de marginación y, lógicamente, una pérdida de calidad de vida respecto a los demás que no sufran esta falta, siendo las personas discapacitadas las que la sufrirán en mayor grado.

\footnotetext{
${ }^{2}$ Alonso López (Coord.). Libro Verde de la Accesibilidad en España. Diagnóstico y bases para un plan integral de supresión de barreras. Madrid: Instituto de Migraciones y Servicios sociales (IMSERSO). $1^{\text {a }}$ Edición, 2002.

3 CCPT (Comisión Central de Coordinación para la Promoción de la Accesibilidad). Concepto Europeo de Accesibilidad. Madrid: Instituto de Migraciones y Servicios Sociales (IMSERSO) - Centro Estatal de Autonomía Personal y Ayudas Técnicas (CEAPAT), p. 35, 1996.
} 
Más allá, el equipo de Expertos Helios ${ }^{4}$, afirma que la falta de accesibilidad puede llegar, en algunos casos, a provocar situaciones de estigmatización, vulnerabilidad, exclusión social y problemas psicológicos a las personas afectadas. La incapacidad de la sociedad para eliminar las barreras de movilidad, de comunicación y de comprensión es sintomática de la atención desigual que merecen las personas con capacidades reducidas.

La accesibilidad es el grado en el que todas las personas pueden utilizar un objeto, visitar un lugar o acceder a un servicio, independientemente de sus capacidades técnicas, cognitivas o físicas.

Para promover y mejorar la accesibilidad, equiparando las posibilidades de todas las personas, podemos hacer uso de las ayudas técnicas como el alfabeto Braille, la lengua de señas, una silla de ruedas, señales auditivas de los semáforos, etc.

Esto supone que un lugar que presenta buenas condiciones de accesibilidad puede recibir a toda clase de gente sin que exista un perjuicio o una dificultad para nadie.

El Libro Blanco de la Accesibilidad ${ }^{5}$ (ACCEPLAN), que fue el borrador del I Plan Nacional de Accesibilidad 2004-2012, tiene como lema en su portada: "Por un nuevo paradigma, el Diseño para Todos, hacia la plena igualdad de oportunidades".

Este lema define y resume perfectamente el espíritu de los términos accesibilidad y Diseño para Todos y su relación con la discapacidad, siendo parte fundamental del planteamiento de este trabajo.

Más allá, el equipo de expertos Helios (Social Integration. Annual Report, 1995) afirma que la falta de accesibilidad puede llegar, en algunos caos a provocar situaciones de estigmatización, vulnerabilidad, exclusión social y problemas psicológicos a las personas afectadas.

La incapacidad de la sociedad para eliminar las barreras de movilidad, de comunicación y de comprensión es sintomática de la atención desigual que merecen las personas con capacidades reducidas o diversidad funcional.

Disponemos de ayudas técnicas como el alfabeto Braille, la lengua de señas, señales auditivas en los semáforos, andadores, sillas de ruedas, bastones, etc., pero nos falta la adaptación a los grandes temas pendiente, la eliminación de barreras arquitectónicas, la adaptación a productos y servicios, viviendas, internet, transportes, edificios, y sobre todo las acciones políticas, administrativas, judiciales, sociales y comunitarias, entre otras para llevar a cabo semejante empresa.

Una de las mayores expresiones de discriminación es la dificultad o imposibilidad de incorporarse al mundo laboral, educativo, de ocio, o a la vida social en general, por la existencia de barreras físicas, de acceso a la comunicación e información $\mathrm{u}$ otras que afectan con especial incidencia a las personas discapacitadas ${ }^{6}$.

Podemos hablar de accesibilidad en el urbanismo, en el transporte, en los edificios y viviendas, en las comunicaciones y en definitiva en cualquier situación,

\footnotetext{
${ }^{4}$ Equipo expertos Helios. Social Integration. Annual Report. Brussels 1995.

5 Alonso López, F. (Coord.) Libro Blanco del Plan de Accesibilidad ACCEPLAN (Borrador). Madrid: Ministerio de Trabajo y Asuntos Sociales - Instituto de Migraciones y Servicios Sociales (IMSERSO). Edición digital: Web de SID, 2003.

${ }^{6}$ Alonso López (Coord.). Libro Verde de la Accesibilidad en España. Diagnóstico y bases para un plan integral de supresión de barreras. Madrid: Instituto de Migraciones y Servicios sociales (IMSERSO). 1를 Edición, 2002.
} 
desde acudir a un teatro con algún tipo de discapacidad, física, psíquica o sensorial, o tratarse simplemente de una persona mayor hasta una persona extranjera que no conoce el idioma.

No podemos olvidarnos de las dificultades que las personas discapacitadas pueden tener en el acceso a la World Wide Web (Internet). La proliferación de las tecnologías de la información y la sociedad del conocimiento, específicamente la información, tiene una relevancia muy significativa como factor de control y poder y a la par como elemento discriminante en cuanto a la estratificación social.

Aquí se ponen de manifiesto multitud de carencias de todo tipo, personas discapacitadas sensoriales, sobre todo donde se hace necesario poner en práctica por parte de los diseñadores y desarrolladores, multitud de soluciones que además no dependan del modelo de equipo o aplicaciones que se empleen para navegar.

"El poder de la Web está en su universalidad. El acceso de todo el mundo con independencia de su discapacidad es un aspecto esencial" [Tim Berners-Lee, inventor de World Wide Web] Presentación en TED 2014. A Magna Carta for the Web.

Aunque en la literatura existen distintas clasificaciones de barreras (véase Smith, 1987 o Amengual, 1996, entre otros), nos centramos en la clasificación que se presenta en el Libro Verde de la Accesibilidad (IMSERSO, 2002) en el que se hace referencia a las principales barreras encontradas por diferentes tipos de personas discapacitadas.

Para las personas discapacitadas físicas:

- Desniveles en aceras, transportes, edificios con escaleras

- $\quad$ Altura de los mostradores, taquillas, cabinas telefónicas, buzones

- $\quad$ Estrechez de pasillos, ascensores, vagones de tren

- $\quad$ Puertas pesadas, giratorias, estrechas, no automáticas

- Aseos no adaptados en edificios y locales públicos

- Pavimentos rotos, nulos o demasiado resaltados

- Obstáculos continuos en la vía pública, fijos o móviles

Para las personas discapacitadas visuales:

- Mala señalización: letra pequeña, no textos en Braille

- Obstáculos continuos en la vía pública, fijos o móviles

- Demasiada gente hace perder sus puntos de referencia

- $\quad$ Pavimentos en mal estado

- Cristales en puertas o en separación de ambientes

- $\quad$ Objetos situados a baja altura: papeleras, ramas de árboles

- $\quad$ Escaleras no señalizadas

Para las personas discapacitadas auditivas: 
- $\quad$ Falta de paneles y carteles informativos

- Comunicación con el personal (no conocen la lengua de signos)

- Ausencia de video portero y avisadores luminosos de timbres

- No hay teléfonos de texto o fax públicos

- Carencia de sistemas de alarma sonoros y luminosos

- $\quad$ Pocas películas y cine subtitulado

En general, barreras de comunicación y acceso a la información. Esto supone que un lugar que presenta buenas condiciones de accesibilidad puede recibir a toda clase de personas usuarias, sin que exista un perjuicio o dificultad de acceso para ninguno de ellos.

Para llegar al cumplimiento de la Accesibilidad Universal podemos tomar como referencia los llamados criterio DALCO, acrónimo utilizado para señalar el cumplimiento de determinadas características que facilitan la Accesibilidad:

- Deambulación: tanto horizontal como vertical. Se refiere a la movilidad, tanto horizontal como vertical; esta movilidad puede ser a través de los medios propios del individuo, como a través de ayudas técnicas (silla de ruedas, muletas, etc.) o mediante el uso de medios de transporte. Una componente fundamental de la movilidad es el estudio de los ACCESOS, ya que la condición primordial para poder disfrutar de un servicio es poder acceder a él, por ello haremos una especial insistencia en este aspecto. Este requisito afecta a los siguientes entornos y situaciones: accesos, anchos de paso, anchos de giro, obstáculos, desniveles verticales: rampas, escaleras, ascensores, etc.

- Aprehensión: de cualquier tipo de objeto, pasamanos, manivelas o mecanismos para abrir las puertas, etc. se refiere a las capacidades de aprehender, alcanzar y agarrar, y engloba no sólo el alcance manual, sino también el alcance auditivo y el visual. Este requisito afecta a los siguientes entornos y situaciones: alcance manual: alcance de botoneras e interruptores, colocación de mecanismos, manillas y herrajes, grifos, pasamanos, asas, barras de apoyo. alcance visual: señalética, elección de colores, tamaño de textos. alcance auditivo: avisos por megafonía, señales de alarma, mostradores de atención al público.

- Localización: de lugares en general, consultas, El usuario debe conocer en cada momento en donde se encuentra y dónde encontrar información para encontrar algo o a alguien. Este concepto engloba y hace referencia a muchos otros como orientación o señalización. Este requisito se refiere a espacios físicos como un edificio, pero también al diseño de interfaces (botoneras, señalética, ...) en las que el usuario ha de ser capaz de encontrar la información que busca.

- $\quad \boldsymbol{C O}$ municación: Entendiendo por comunicación los procesos de emitir, recibir e intercambiar información a través de distintos canales: oral, escrito, visual, auditivo. En este apartado estaría incluido todo lo referente a la señalética y a la cartelería, así como todos los sistemas de aviso, alarmas, megafonía, señalización táctil, folletos, planos, interfaces. Entornos especialmente afectados por este requisito: zonas de atención al público, mostradores o taquillas. 
Estos requisitos están relacionados entre sí y aparecen, en distinta medida, en todos los entornos y elementos: por ejemplo, en el diseño de una puerta encontramos requisitos de deambulación (dimensiones de la puerta) de aprehensión (diseño de la manilla) de localización (el usuario debe saber a donde lleva esa puerta antes de cruzarla) y de comunicación (el cartel de la puerta debe ser entendible)

\section{Propuestas y discusión sobre la Discapacidad y la Accesibilidad Universal. La realidad actual}

Entonces, ¿qué aporta la Accesibilidad Universal a la mejora de la discapacidad? ¿Cuál es la situación actual en cuanto a despliegue de medios y recursos para la mejora de la Accesibilidad universal?

Aunque nos encontramos con que la implantación progresiva de las normas de Accesibilidad debido al cumplimiento lento de la legislación y normativas vigentes, nos posibilitarán la mejora de la justicia social y la calidad de vida de millones de personas, la principal excusa desde las administraciones públicas suele ser la falta de presupuesto, sin embargo es erróneo este planteamiento ya que existen numerosos estudios que ponen claramente de manifiesto que las mejoras que se produzcan en torno a la accesibilidad a edificios, lugares de ocio, transportes, internet, marketing, etc., además de facilitar la calidad de vida a los casi 12 millones de usuarios entre personas discapacitadas permanentes y temporales y mayores, dan además auténticos frutos en la economía de un país.

"Facilitar un entorno accesible significa procurar una mayor autonomía personal, permitir una vida independiente y de ciudadanía plena, pero también reducir la necesidad de institucionalización y de ayuda familiar. Por todo ello es indiscutible que la disminución de las barreras significa el aumento del nivel de bienestar". (Olivera, 2006)

Entendemos que no se pone en duda la enorme necesidad de mejorar la Accesibilidad, no obstante, se observa a muchos niveles un escaso seguimiento de buena parte de normas y leyes en este sentido, ya sea en lo referente a salud (López Cala, 2011), edificios públicos y administrativos (Huerta Peralta, 2006), transportes (Vega, P., Alonso, F. 2006), etc. Llevándonos a concluir que al parecer la necesidad de esas mejoras no es unánime o es más complicada de aplicar de lo que se suponía en principio, bien sea por falta de interés, de presupuesto, complejidad, desconocimiento, prejuicios, etc.

Un claro ejemplo de la necesidad e importancia en la mejora de la Accesibilidad es el proyecto ISEMOA ${ }^{7}$ (2010-2013), cofinanciado por la Unión europea, donde pone de manifiesto que las políticas y medidas destinadas a mejorar los entornos accesibles benefician a todos, no solo a las personas discapacitadas.

En lo referente por ejemplo a la mejora en los transportes públicos, reduciría la dependencia del automóvil y una movilidad de manera autónoma para aquellas personas que no tienen acceso a los coches. Además, mejoraría el tráfico motorizado en general,

\footnotetext{
${ }^{7}$ http://www.isemoa.eu/docs/42/ISEMOA_benefit_brochure_ES_screen_2.pdf
} 
mejoraría la salud, habría una mayor inclusión social, un aumento de la calidad de vida, ahorro de costes, menor contaminación, ahorro de energía, etc.

En lo referente al consumo como otro ejemplo, la mejora de la accesibilidad según Luis Casado (2016), acuña tres grandes ideas:

- La igualdad de oportunidades y la no discriminación a lo que todos tenemos derecho también ha de aplicarse al ámbito del consumo diario.

- El mercado de personas con diversidad funcional y sus familiares supone en España un volumen de ventas de más de 13.000 millones de euros. Lo cual significa que existe una enorme oportunidad de negocio en la mejora de la accesibilidad de los 3,2 millones de hogares en España con al menos una persona con discapacidad.

- La legislación europea, nacional y autonómica apunta a una mayor inclusión social, con lo que no es una estrategia inteligente esperar a que la ley obligue a set accesibles teniendo en cuenta la gran población discapacitada, dependiente y mayor existente.

Las políticas sociales, los Libros Blancos, los grandes estudios y documentos, la legislación y normas sobre determinadas materias y conceptos y las tan frecuentes reuniones, charlas, conferencias, congresos, etc., no llegan a ser suficientes para la implementación real y operativa de soluciones que mejoren de forma efectiva los términos a los que nos referimos en este trabajo, como es la Discapacidad y la Accesibilidad Universal.

Cada día podemos observar que efectivamente hay ciertas mejoras en los transportes, edificios, lugares de ocio y cultura, supermercados y grandes superficies, y un largo etc., pero no son suficientes y vemos como tras años de estar legisladas adecuadamente y de forma modélica las normas que deben imperar a nivel de nuestra sociedad, siguen sin cumplirse.

Esta falta de cumplimiento tiene numerosas razones de ser, a saber, por la mentalidad de nuestros gestores que ven estos conceptos como etéreos o necesarios, pero a largo plazo. Por falta de interés en entender y comprender lo importante que es para millones de personas usuarias. O simplemente que es lo que más se utiliza como excusa, por falta o escaso presupuesto.

Luego, ¿para cuándo entonces la verdadera implementación de las soluciones destinadas a estos conceptos?

Finalmente, llegamos a pensar que son los gestores y responsables políticos los que tienen de alguna forma las soluciones tangibles para llevar a cabo esta empresa, pero olvidamos que todos somos partícipes también de estas mejoras y soluciones, con nuestro conocimiento, actitudes y aptitudes, solidaridad, transmisión de la información para una mejor concienciación social y desde luego algo que falta hoy en día cada vez más, y nos referimos al altruismo y al voluntarismo.

Si empezamos con propuestas a todos los niveles, es decir, desde las asociaciones de barrio, los ayuntamientos, las Diputaciones y organismos provinciales, luego autonómicos, nacionales e internacionales. Tendremos más oportunidades de que se cumplan las normas y leyes. Pero desgraciadamente tienen que existir mecanismos de control muy estrictos y claros, además de comisiones, observatorios y cualesquiera otros recursos humanos que vigilen la puesta en marcha, seguimiento, control, evaluaciones y 
mejoras constantes de las propuestas que se lleven a cabo en la mejora de las diversas situaciones sobre Discapacidad y Accesibilidad Universal.

Por ejemplo, desde la Atención Primaria de Salud, tenemos una excelente vía de comunicación con la ciudadanía, pues es la entrada al sistema sanitarios de cualquier tipo de discapacidad conocida, con lo cual tenemos aquí una vía importante de información y puesta en marcha de programas, proyectos y planes dirigidos a la población sobre los términos referidos.

Además, se pueden llevar a cabo numerosas acciones e intervenciones, como la información en forma de campañas divulgativas, folletos, dípticos y trípticos, publicidad televisiva y radiofónica, etc., para informar, enseñar a facilitar la calidad de vida a las personas usuarias discapacitadas y con necesidad de una mejora en la Accesibilidad Universal.

Otro pilar fundamental sería nuestro Sistema Educativo, desde primaria hasta la universidad. La educación sobre estos y otros muchos términos y conceptos está absolutamente abandonada, salvo en honrosas excepciones y es en estos lugares y foros donde se hace fundamental y desde edades tempranas el conocimiento extenso sobre la importancia de estos y otros muchos temas.

Las materias que se imparten durante la etapa educativa te dan conocimientos más o menos efectivos y específicos, pero perdemos la ocasión de formar también a nuestras próximas generaciones en temas como los que tratamos y otros muchos que formarían en mejor grado las conciencias y la calidad humana y social de los jóvenes que van a ser quienes al fin y al cabo tengan la oportunidad de cambiar nuestra ciudad, país o resto del mundo. Con sus ideas, con sus conocimientos, con sus voluntades sumadas y con la lucha por la igualdad y la solidaridad para con quienes la sociedad no cumpla.

\section{Discusión y conclusiones}

España es un país con un modelo de atención y bienestar social basado en los derechos humanos, cuyo objetivo entre otros es la no discriminación y la igualdad de oportunidades.

Tradicionalmente las personas con discapacidad han sido sujetos de prejuicios, encontrándose en muchas circunstancias en situación de vulnerabilidad social, sufriendo discriminación y exclusión con una disminución en su calidad de vida y con problemas para acceder a determinados servicios, productos y lugares.

Por lo tanto, creemos que es fundamental tener en cuenta que hay que mejorar constantemente y de forma inmediata y exponencial, la situación de los entornos productos y servicios destinados a combatir o paliar de alguna manera los distintos grados de discapacidad. Y esa mejora puede aparecer desde la aplicación y desarrollo del Concepto Accesibilidad Universal.

Es necesario por tanto reflexionar sobre si nuestra sociedad está siendo capaz de desarrollar y realizar acciones e intervenciones administrativas, legislativas, políticas, económicas, educativas y de información y concienciación social en lo concerniente a la Accesibilidad Universal que aseguren el mantenimiento de los derechos de estas personas usuarias. 
Por otra parte, las deficiencias de nuestra sociedad y la falta de solidaridad e implementación en la puesta en marcha y mantenimiento de las políticas orientadas hacia las personas con diversidad funcional es a veces un importante hándicap para el correcto cuidado de estos.

Esto hace que nuestra sociedad no aparezca como justa, equitativa, global, responsable y garante de los derechos fundamentales de todos los ciudadanos por igual ante cualquier tipo de discapacidad, ya sea temporal o permanente.

Nuestra obligación como personas, miembros de una sociedad, intelectuales, investigadores y cualesquiera otros interesados en mejorar nuestro sistema, que de alguna forma trabaja cada día para ser más justo, es establecer soluciones en forma de políticas, intervenciones y mecanismos sobre las personas, productos, servicios y entornos.

Debemos realizar aportaciones estratégicas referidas a la mejora de la calidad de vida sobre todas las personas, pero más aún sobre aquellas discapacitadas que no llegan a tener las mismas oportunidades frente al acceso a entornos, productos y servicios y frente a los derechos constitucionales y legales. Pensemos por ello que vivimos por tanto en una sociedad permanentemente injusta.

De la misma forma, la realidad social percibida en España y otros muchos países del mundo es que seguimos encontrándonos con diversas situaciones de falta de accesibilidad de un número importante de personas usuarias a los lugares de uso cotidiano formal e informal, laboral o de ocio, de necesidad diaria u ocasional. Desde la entrada a un museo, hospital, centro de salud, transporte público y un gran número de establecimientos por poner ejemplos, observamos cada día situaciones de inaccesibilidad.

Podemos considerar como puntos fuertes en lo referente a la situación de la Accesibilidad Universal y el Diseño para Todos, así como en la implementación de soluciones y mejora de las situaciones de Discapacidad en general, la amplia, profusa y excelente legislación y normativa actual.

En cuanto a los puntos débiles, consideraríamos la escasa o casi inexistente, en ocasiones asignación presupuestaria y la lentitud para la puesta en marcha de la mejora de estos temas fundamentales, la Accesibilidad Universal y la Discapacidad en España.

Para ello debemos conseguir entre lo institucional y no institucional trazar metas alcanzables y factibles, con un ritmo mucho mayor de lo que se viene haciendo y de esta forma, quizás consigamos solucionar o paliar en gran medida estas situaciones, como decimos, de auténtico agravio comparativo y de incumplimiento social de los derechos fundamentales de las personas.

Por lo tanto, hay que trabajar con la máxima sensibilidad, poniendo no solo la intención y la vista en las futuras acciones y políticas de la administración, sino realizar una labor de información, control, concienciación, y mejora de la calidad humana, en definitiva.

\section{Referencias}

López, F.A. (2002). Libro Verde de la Accesibilidad en España. Diagnóstico y bases para un plan integral de supresión de barreras. Madrid: IMSERSO. Recuperado de http://sid.usal.es/idocs/F8/8.1-5999/libro_verde_accesibilidad.pdf 
Casado, L. (2016). Cómo mejorar la Accesibilidad Universal en el Gran Consumo: Una herramienta de mejora competitiva y social. Recuperado de http://www.camaranavarra.com/content/userfiles/file/pdfs/Guia_Accesibilidad_ mercado_consumo.pdf

Código Técnico de la Edificación, Documento Básico de Seguridad de Utilización y Accesibilidad (DB-SUA-9).

CCPT-Comisión Central de Coordinación para la Promoción de la Accesibilidad (1996). Concepto Europeo de Accesibilidad. Madrid: Instituto de Migraciones y Servicios Sociales (IMSERSO) - Centro Estatal de Autonomía Personal y Ayudas Técnicas (CEAPAT), p. 35.

Comunicación de la Comisión al Consejo, al Parlamento Europeo, al Comité económico y social y al Comité de las Regiones. Hacia una Europa sin barreras para las personas con discapacidad (COM 2000-284 final).

Decreto 72/92 de 5 de mayo, por el que se aprueban las normas técnicas para la accesibilidad y la eliminación de barreras arquitectónicas y en el transporte de Andalucía.

Decreto 133/92, de 21 de julio, por el que se establece el régimen transitorio en la aplicación del decreto 72/92, de 5 de mayo, por el que se aprueban las normas técnicas para la accesibilidad y la eliminación de barreras arquitectónicas.

Directiva 2000/78/CE del Consejo de 27 de noviembre de 2000 relativa al establecimiento de un marco general para la igualdad de trato en el empleo y la ocupación.

Decreto 293/2009, de 7 de julio, por el que se aprueba el reglamento que regula las normas para la accesibilidad en las infraestructuras, el urbanismo, la edificación y el transporte en Andalucía.

Equipo expertos Helios (1995). Social Integration. Annual Report. Bruselas.

Geiser.P., Chervin, P., (2013) Handicap Internacional. Guía sobre Discapacidad y Desarrollo. Recuperada de http://www.cocemfe.es/cooperacion/images/pdf/Guia_discapacidad_y_desarroll o_COCEMFE.pdf

Huerta, J. (2006) Discapacidad y Accesibilidad. La Dimensión desconocida. Comisión especial de estudio sobre discapacidad del congreso de la República del Perú. Recuperado de http://bvs.minsa.gob.pe/local/minsa/920_GOB422.pdf

Informe Mundial sobre la Discapacidad (2011). Organización Mundial de la Salud. Ed. OMS.

Jefatura del Estado. BOE núm. 299, de 15 de diciembre de 2006. Ley 39/2006, de 14 de diciembre, de Promoción de la Autonomía Personal y Atención a las personas en situación de dependencia (Art. 2).

La Constitución Española de 1978. (Castellano)

Ley de Integración Social del Minusválido LISMI (B.O.E. 103, 30 de abril de 1982).

Ley 51/2003 de 2 de diciembre sobre Igualdad de Oportunidades No Discriminación y Accesibilidad Universal. (LIONDAU) B.O.E. 289, 3 de diciembre de 2003. 
Ley 3/90 por el que se modifica la Ley 49/60 de propiedad horizontal, para facilitar la adopción de acuerdos que tengan por finalidad la adecuada habitabilidad de minusválidos en el edificio de su vivienda (BOE 22/06/90).

Ley 29794 de Arrendamientos Urbanos (BOE 25/11/94).

Ley 15/95 sobre límites del dominio sobre inmuebles para eliminar barreras arquitectónicas a las personas con discapacidad (BOE 31/05/95).

Ley 8/99 de Reforma de la Ley 49/60 de 21 de julio sobre Propiedad Horizontal (BOE 08/04/99).

Ley 38/99 de Ordenación de la Edificación (BOE 06/11/99).

Ley 27/2007, de 23 de octubre, por la que se reconocen las lenguas de signos españolas y se regulan los medios de apoyo a la comunicación oral de las personas sordas, con discapacidad auditiva y sordo ciegas (BOE 24/10/07).

Ley 26/2011, de 1 de agosto, de adaptación normativa a la Convención Internacional sobre los Derechos de las Personas con Discapacidad (BOE 2/08/11).

Ley 8/2013, de 26 de junio, de rehabilitación, regeneración y renovación urbanas (BOE 27/06/13).

Ley 5/98 de 23 de noviembre, relativa al uso en Andalucía de perros guía por personas con disfunciones visuales.

López Cala, G. (2011). La Accesibilidad en el Sistema Sanitario Público Andaluz. El caso del Distrito Sanitario de Jaén. Tesis Doctoral, Universidad de Jaén. http://hdl.handle.net/10953/540

Muñoz, A.P. (2010). Discapacidad: contexto, concepto y modelos. Internacional Law. Revista colombiana de Derecho internacional, 381-414.

Normas Uniformes sobre la Igualdad de Oportunidades para las personas con discapacidad. Resolución de las Naciones Unidas. Aprobada por la Asamblea General. Cuadragésimo octavo período de sesiones, 20 de diciembre de 1993.

Olivera Poll, A. (2006). Discapacidad, Accesibilidad y espacio excluyente. Una perspectiva desde la Geografía Social Urbana. Treballs de la Societat Catalana de Geografía, 61/62, 326-343.

OMS (2001). Clasificación Internacional del Funcionamiento, de la Discapacidad y de la Salud. Madrid: IMSERSO.

Orden 3/95, de 3 de mayo de 1995, por la que se crea una comisión técnica dependiente de la comisión de accesibilidad y eliminación de barreras arquitectónicas, urbanísticas y del transporte en Andalucía.

Orden 5/96, de 5 de septiembre de 1996, por la que se aprueba el modelo de ficha para la justificación del cumplimiento del Decreto 72/1992, de 5 de mayo, de la Consejería de la Presidencia de la Junta de Andalucía.

Orden de 18 de enero de 2002, de la Consejería de Asuntos Sociales, por la que se aprueba el modelo y procedimiento de concesión de la tarjeta de aparcamiento de vehículos para Personas con movilidad reducida (BOJA n ${ }^{\circ} 18$, de 12 de febrero de 2002). 
Orden del Ministerio de Obras Públicas y Urbanismo sobre las características de los accesos, aparatos elevadores y condiciones interiores de las viviendas para minusválidos proyectadas en inmuebles de protección oficial (BOE 18/03/80).

Orden del Ministerio de Educación y Ciencia por la que se aprueban los programas de necesidades para la redacción de los proyectos de construcción y adaptación de centros de educación especial (BOE 06/04/81).

Orden del Ministerio de Obras Públicas y Urbanismo por la que se regulan las condiciones de tramitación de las ayudas de protección a la rehabilitación de viviendas (BOE 25/11/83).

Orden del Ministerio de Industria, Comercio y Turismo por la que se modifica la instrucción técnica complementaria MIE-AEM 1 del Reglamento de Aparatos de Elevación y Manutención (BOE 17/09/91).

Orden CTE/3191/2002, de 5 de diciembre, por la que se tipifican nuevas reformas de importancia y se modifican los anexos I y II del Real Decreto 736/1988, de 8 de julio, por el que se regula la tramitación de reformas de importancia de vehículos de carretera y se modifica el artículo 252 del Código de la Circulación (BOE n ${ }^{\circ}$ 301, 17 de diciembre de 2002).

Orden VIV/561/2010, de 1 de febrero, por la que se desarrolla el documento técnico de condiciones de accesibilidad y no discriminación para el acceso y utilización de los espacios públicos urbanizados (BOE 11/03/10).

Resolución del Consejo de 6 de febrero de 2003 sobre Accesibilidad Electrónica.

Resolución B4/0985/98 del Parlamento Europeo, sobre el lenguaje mímico. Consultation Document European Accessibility

Real Decreto $2159 / 78$ por el que se aprueba el reglamento de planeamiento para el desarrollo y aplicación de la ley sobre régimen del suelo y ordenación urbana (BOE 15/09/78).

Real Decreto 355/80 de 25 de enero sobre reserva y situación de las viviendas de protección oficial destinadas a minusválidos (BOE 28/02/80).

Real Decreto 248/81 sobre medidas de distribución de la reserva de viviendas destinadas a minusválidos establecidas en el RD 355/80 de 25 de enero (BOE 26/10/81).

Real Decreto 1634/83 por el que se establecen normas de clasificación de los establecimientos hoteleros (BOE 17/06/83).

Real Decreto 2329/83 sobre protección a la rehabilitación del patrimonio residencial y urbano (BOE 07/09/83).

Real Decreto 3250/83 por el que se regula el uso de perros guía para deficientes visuales (BOE 02/01/84).

Real Decreto 556/89 por el que se arbitran medidas mínimas sobre accesibilidad en los edificios públicos (BOE 31/05/89).

Real Decreto 1314/1997, de 1 de agosto, por el que se dictan las disposiciones de aplicación de la Directiva del Parlamento Europeo y del Consejo 95/16/CE, sobre ascensores. 
Real Decreto 1/2002 de 11 de enero, sobre medidas de financiación de actuaciones protegidas en materia de vivienda y suelo del Plan 2002-2005 (BOE 12/01/02).

Real Decreto 505/2007, de 20 de abril, por el que se aprueban las condiciones básicas de accesibilidad y no discriminación de las personas con discapacidad para el acceso y utilización de los espacios públicos urbanizados y edificaciones (BOE 11/05/07).

Real Decreto 1494/2007, de 12 de noviembre, por el que se aprueba el Reglamento sobre las condiciones básicas para el acceso de las personas con discapacidad a las tecnologías, productos y servicios relacionados con la sociedad de la información y medios de comunicación social (BOE 21/11/07).

Real Decreto 1544/2007, de 23 de noviembre, por el que se regulan las condiciones básicas de accesibilidad y no discriminación para el acceso y utilización de los modos de transporte para personas con discapacidad (BOE 4/12/07).

Real Decreto 422/2011, de 25 de marzo, por el que se aprueba el Reglamento sobre las Condiciones Básicas para la participación de las personas con discapacidad en la vida política y en los procesos electorales.

Real Decreto-ley 8/2011, de 1 de julio, de medidas de apoyo a los deudores hipotecarios, de control del gasto público y cancelación de deudas con empresas y autónomos controladas por las entidades locales, de fomento de la actividad empresarial e impulso de la rehabilitación y de simplificación normativa. (BOE 7/07/11).

Real Decreto Legislativo 1/2013, de 29 de noviembre, por el que se aprueba el Texto Refundido de la Ley General de los derechos de las personas con discapacidad y de su inclusión social (BOE 3/12/2013).

Vega, P., Alonso, F. (2006) La accesibilidad del transporte en autobús. Diagnóstico y soluciones. Ministerio de Trabajo y Asuntos Sociales. Madrid: IMSERSO.

Web Cruz Roja Española. Consultado en enero de 2017. www.cruzroja.es

Web de Plusesmas. Consultada en febrero de 2017. www.plusesmas.com

Fecha de recepción: 12/10/2017

Fecha de revisión: 31/10/2017

Fecha de aceptación: 17/03/2018 
